Article

\title{
Hydrophobic and Icephobic Behaviour of Polyurethane-Based Nanocomposite Coatings
}

\author{
Bartlomiej Przybyszewski ${ }^{1, *}{ }^{\mathbb{D}}$, Anna Boczkowska ${ }^{1} \mathbb{D}$, Rafal Kozera ${ }^{2}$, Julio Mora ${ }^{3}$, \\ Paloma Garcia ${ }^{4}$, Alina Aguero ${ }^{4}$ and Ana Borras ${ }^{5}$ \\ 1 Faculty of Materials Science and Engineering, Warsaw University of Technology, 141 Woloska Str., \\ 02-507 Warsaw, Poland; anna.boczkowska@pw.edu.pl \\ 2 Technology Partners Foundation, 5A Pawinskiego Str., 02-106 Warsaw, Poland; \\ rafal.kozera@technologypartners.pl \\ 3 Ingeniería de Sistemas para la Defensa de España SA, Beatriz de Bobadilla No. 3, 28040 Madrid, Spain; \\ modsurf4.pers_externo@inta.es \\ 4 Instituto Nacional de Técnica Aeroespacial, Área de Materiales Metálicos, Ctra. Ajalvir Km 4, 28850 Torrejón \\ de Ardoz, Spain; garciagp@inta.es (P.G.); agueroba@inta.es (A.A.) \\ 5 Instituto de Ciencia de Materiales de Sevilla (CSIC-US), Americo Vespucio 49, 41092 Seville, Spain; \\ anaisabel.borras@icmse.csic.es \\ * Correspondence: bartlomiej.przybyszewski.dokt@pw.edu.pl; Tel.: +48-234-57-13
}

Received: 29 October 2019; Accepted: 28 November 2019; Published: 2 December 2019

\begin{abstract}
In this paper, hydrophobic nanocomposite coatings based on polyurethane (PUR) modified by nano-silica and silane-based compounds were manufactured by spraying. The main challenge was to assess and improve the hydrophobic as well as anti-icing properties of initially hydrophilic polymer coatings. The prepared nanocomposite coatings were characterized by means of scanning electron microscopy (SEM), optical profilometry and X-ray photoelectron spectroscopy (XPS). The results obtained showed that in order to achieve hydrophobicity, appropriate amounts of nano-silica must be incorporated in the coating, and complete coverage by nano-silica particles is necessary for achieving hydrophobicity. Coating adhesion and the durability of the hydrophobic behaviour were also studied by scratch test and frosting/defrosting cycles, respectively. The results show that use of both nano-silica and silane-based compounds improve the hydrophobic and anti-icing properties of the coating as compared to a non-modified PUR topcoat. A synergistic effect of both additives was observed. It was also found that the anti-icing behaviour does not necessarily correlate with surface roughness and the materials' wetting properties.
\end{abstract}

Keywords: icephobicity; polyurethane; nano-silica; nanocomposite coating; hydrophobicity

\section{Introduction}

Flying into adverse weather conditions is a critical operational issue for all types of aircraft (airplanes, helicopters, drones or unmanned aerial vehicles, etc.). Icing clouds lead to potentially dangerous ice accretion on key elements such as fan and helicopter blades, winglets or slats, etc. by causing changes in the aerodynamic profiles as well as performance degradation due to a decrease in lift and increased drag, torques or vibrations [1-5].

Based on the European Union Aviation Safety Agency (EASA) [6] annual reports, icing in flight remains one of the three largest issues for aviation safety based on the past performance of the system by counting high-risk occurrences or the number of fatalities or through the aggregated risk score. The authors of the report indicated that between the 2012 and 2016, 277 in-flight icing incidents were reported. Six of them were classified as serious incidents and one as fatal. Moreover, in the same 
period, 116 additional on-ground incidents leading to flights delays were reported. These numbers show that icing is dangerous for aviation safety and still common.

The ice protection systems currently used are based on electro-thermal systems that operate once the ice has been formed (de-icing): they melt the ice layer in contact with the solid surface and allow ice removal by aerodynamic forces. However, these methods present several drawbacks: for instance, such systems usually cover only the leading edge of the blade where ice accretes, leading to the potential formation of the so-called "runback ice", caused by liquid water flowing in the aft direction [7]. In addition, these systems operate cyclically (to avoid huge power consumption) allowing ice to accrete up to a thickness of several millimetres prior to removal. Finally, they require heavy devices, as well as high power inputs and generate large quantities of heat which is inappropriate for currently used polymer-based topcoat materials [8].

The aforementioned drawbacks have forced manufacturers and scientists to develop new systems to prevent or mitigate ice formation on aircraft. An alternative is provided by surface coatings-a passive, reliable approach which results in low energy consumption, low costs, does not require expensive equipment, and can confer permanent protection against ice $[9,10]$. The state-of-the-art describes two main coating design strategies to prevent ice accretion. The first is based on superhydrophobic surfaces with very low water adhesion forces and surface-free energies [11,12], whilst the second one is based on low ice adhesion [13]. There are coatings which exhibit both superhydrophobicity and low adhesion which can be synergic [14]. This can usually be achieved by controlling the surface roughening and by employing low surface-energy materials.

Preparation of dual-scale, hierarchical structures inspired by nature (e.g., lotus leaf) has been already widely described in the literature [15-17]. Most of them only concern hydrophobic/superhydrophobic properties of polyurethane (PUR) achieved by the addition of nanoparticles. However, there are no reports on achieving hydrophobic and icephobic properties of PUR using both nanoparticles and silane-based modifiers. This paper presents investigation of such hybrid modification of polyurethane coatings.

Polymer nanocomposites are currently one of the most interesting areas for research and development (R\&D), especially in demanding sectors such as the automotive industry or aviation [18]. Nanoparticles are now widely used for surface roughness modification because of their unique properties, easier processing and commercial availability. Their proper modification and distribution in polymers makes it possible to influence the wettability of the coating $[19,20]$.

Polyurethane is one of the most commonly utilized polymer in different industrial applications, including coatings. Due to very good ultraviolet (UV) resistance and adhesion to aluminium, the PUR topcoats are attractive in aviation, automotive and many others sectors [21,22]. However, PUR macromolecules contain polar groups, which gradually migrate onto the top of the coating and thus interact with the water droplets causing a decrease of hydrophobicity [23]. Because of the fact that polyurethane is a rather hydrophilic material, it is still challenging to manufacture hydrophobic PUR-based coatings with high durability, especially under low temperatures (below freezing). Hence, appropriate modifications of PUR are needed to obtain stable, anti-icing coatings. In this paper, simple volumetric modification techniques have been utilized to modify the chemical composition and microstructure of PUR coatings by adding Si rich compounds and nano-silica particles. The additional objective was to determine nano-silica concentration resulting in improved anti-icing behaviour of investigated material without deterioration of adhesion to the substrate. A synergic effect of both modifications was evaluated.

\section{Materials and Methods}

\subsection{Materials}

A 3 component PUR topcoat (Aviox ${ }^{\circledR}$ finish 77702) was obtained from Akzo Nobel, Netherlands. It was used in combination with the Aerodur ${ }^{\circledR}$ HS 370922 (Akzo Nobel, Sassenheim, Netherlands) 
component amine cured epoxy primer. To modify the topcoat, DC88, a silane/siloxane blend (Dow Corning ${ }^{\circledR}$, Midland, MI, USA) was utilized. The hydrophobic nano-silica Aerosil ${ }^{\circledR}$ R8200 (Evonik, Hanau, Germany) used in this study has a specific surface area of 135-185 $\mathrm{m}^{2} / \mathrm{g}$ and a nominal particle size of $16 \mathrm{~nm}$. It was produced by treating nano-silica with bis(trimethylsilyl)amine $\left(\mathrm{C}_{6} \mathrm{H}_{19} \mathrm{NSi}_{2}\right)$. All these materials were used as received. The modified PUR coatings were deposited on 2024 aluminium alloy (WMH Group, Essen, Germany).

\subsection{Preparation and Deposition of Silica/Polyurethane (PUR) Nanocomposite Coatings}

The 2024 aluminium alloy coupons were first cleaned in an ultrasonic acetone bath for $15 \mathrm{~min}$. Initially, both primer components were mixed according to the manufacturer's indications, then sprayed on Al coupons by means of a Walther ${ }^{\circledR}$ Pilot XIII air spray gun (Wuppertal-Vohwinkel, Germany) and left to dry for $3 \mathrm{~h}$. Meanwhile, PUR topcoats with different content of nano-silica (1 wt \% and 5 wt \%) and DC88 (5 wt \%) were prepared by mixing the liquid additive with PUR using a magnetic stirrer. Before spraying, nano-silica suspensions on the modified PUR coating were prepared by means of an ultrasonic gun (VCX 750, Sonics and Materials Inc., Newtown, CT, USA) for 30 min. The suspensions were then sprayed on top of the already applied primer and left to dry for $24 \mathrm{~h}$. The different compositions prepared are shown in Table 1.

Table 1. List of manufactured polyurethane (PUR) nanocomposite coatings.

\begin{tabular}{ccc}
\hline Sample & $\mathrm{SiO}_{\mathbf{2}}$ Content [wt \%] & DC88 Content [wt \%] \\
\hline ref & - & - \\
1 & 1 & - \\
2 & 5 & - \\
3 & - & 5 \\
4 & 5 & 5 \\
\hline
\end{tabular}

\subsection{Characterization}

The coating thickness was measured by an Extech ${ }^{\circledR}$ (Nashua, NH, USA) coating thickness tester, at least six times across the sample using the eddy current measurement mode.

Roughness ( $R$ a and $R z$ ) was measured by means of an optical profilometer (Wyko NT9300, provided by Veeco, Plainview, NY, USA). All the measurements were acquired on a $450 \mu \mathrm{m} \times 350 \mu \mathrm{m}$ area. 6 different, randomly selected areas were measured on each sample. Within one location, 10 measurements of $400 \mu \mathrm{m}$ were carried out.

The microstructure of the coating surfaces was observed with a scanning electron microscope (SEM, Hitachi S5500, Tokyo, Japan) operated at $5 \mathrm{kV}$. All samples were sputtered with a gold-palladium coating.

The chemical composition of the surface layer on the investigated materials was analysed using X-ray photoelectron spectroscopy (XPS). Samples were characterized in a DLSEGD-Phoibos-Hsa3500 from SPECS (Berlin, Germany) with non-monochromatic Al K-alpha radiation (hv = $1486.6 \mathrm{eV})$ as the excitation source and working in the pass energy constant mode. Pass energy for general spectra was settled at $50 \mathrm{eV}$ and for high-resolution peaks at $35 \mathrm{eV}$. Binding energy scale of the spectra was calibrated to the C1s signal taken at $284.5 \mathrm{eV}$.

Wettability properties (static contact angle (SCA), advancing contact angle (ACA), receding contact angle (RCA), sliding angle (SA)) were measured by a contact angle measurement system (Data Physics GmbH OCA 15, Filderstadt, Germany). All angles of each sample were measured at least three times across the sample surface using the sessile drop method, by dispensing $3 \mu \mathrm{L}$ (SCA), $5 \mu \mathrm{L}$ (ACA, RCA) and $10 \mu \mathrm{L}$ (SA) of deionized water on the sample's surface. The contact angle hysteresis (CAH) was calculated as the difference between ACA and RCA.

Frosting/defrosting cycles were performed to assess the durability of the investigated coatings. The samples were cooled to $-20{ }^{\circ} \mathrm{C}$ by placing them in a freezing chamber for $24 \mathrm{~h}$. The samples were then extracted until a temperature of $25^{\circ} \mathrm{C}$ was reached. Water droplets resulting from thawing of 
frost formed on the samples' surface were removed manually by water-absorbing paper followed by air drying at room temperature for $24 \mathrm{~h}$. This procedure was repeated 10 times. After each frosting/defrosting cycle, the wetting properties of the coating (SCA) were measured.

The coating system adhesion to the $\mathrm{Al}$ alloy substrate was measured by the Revetest scratch tester (CSM Instruments, Peseux, Switzerland) with a displacement speed of $10 \mathrm{~mm} / \mathrm{min}$ and a maximum force of $20 \mathrm{~N}$. The scratch length was $10 \mathrm{~mm}$. A steel ball indenter $(\mathrm{R}=0.2 \mathrm{~mm})$ was used.

To evaluate the ice accretion, a simple screening test was used. It allows for measurement of the mass of accreted ice, and for comparing the icing behaviour of different coatings. The test procedure is as follows: (1). the cleaned and dried sample is weighed before the test, inside a zip lock plastic bag, (2). a thermocouple is attached to the upper side of the sample (far of the testing zone), (3). the sample is placed inside a freezing chamber (Climas, mod. $\mathrm{CH} / 300 / \mathrm{AF} / 30$ ) and cooled to $-10^{\circ} \mathrm{C}$, (4). when the sample reaches $-10^{\circ} \mathrm{C}$, air is flown at $60 \mathrm{~m} / \mathrm{s}$ through a nozzle located at $45^{\circ}$ from the sample's surface at a distance of $3 \mathrm{~cm}$ (see Figure 1).

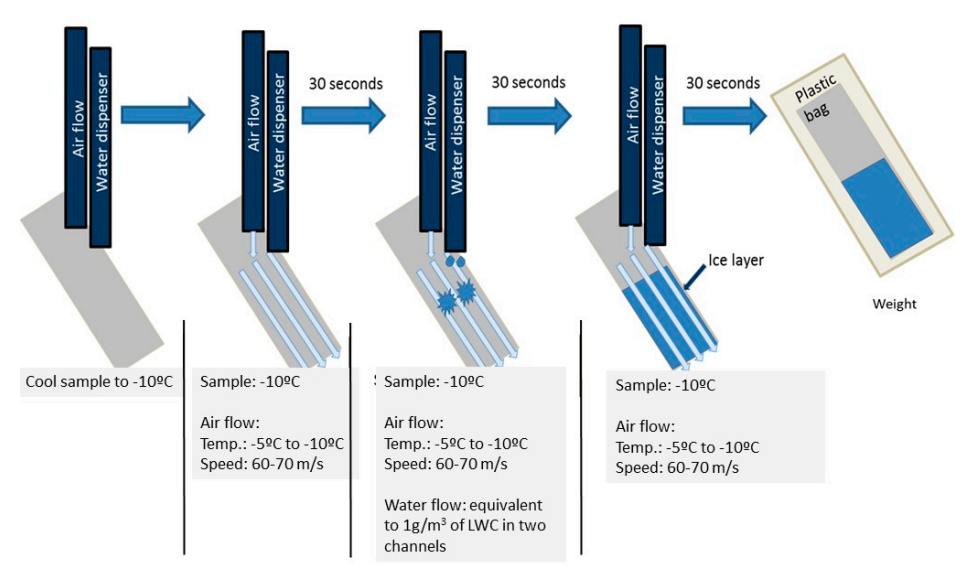

Figure 1. Scheme of the procedure for testing in ice accretion screening.

The air speed was measured by means of a Pitot probe PCE-PFM 2 (PCE Instruments, Southampton, UK) prior to carrying out the tests, (5). after $30 \mathrm{~s}$ under air flow, supercooled water droplets begin to fall from two droppers (internal diameter $-1 \mathrm{~mm}$ ) located at a distance of $3 \mathrm{~cm}$ at $45^{\circ}$ from the sample's surface (Figure 1). The water is fed by means of a peristaltic pump (Gilson Minipuls 3) at 2.5 $\mathrm{mL} / \mathrm{min}$ for $30 \mathrm{~s}$. The water droplets' diameter is $2 \mathrm{~mm}$ but the air flow breaks them so they become significantly smaller, (6). After $30 \mathrm{~s}$, the water flow is stopped and the sample is left with the cooled air flow for another $30 \mathrm{~s},(7)$. The sample is then immediately placed in the same plastic bag to be weighed. The water flow was controlled to have a liquid water content (LWC) of $1 \mathrm{~g} / \mathrm{m}^{3}$ which is typical of glace ice formation. The following calculation was performed:

$$
\operatorname{LWC}\left(\frac{g}{m^{3}}\right)=\frac{\text { Peristaltic water flow }\left(\frac{g}{s}\right)}{\text { Exposed sample area }\left(m^{2}\right) \times \text { Air velocity }\left(\frac{m}{s}\right)}
$$

Each sample was tested at least three times to obtain a mean value with its respective deviation. The ice accretion was evaluated by direct weight measurement (difference before and after the test). The mean value of all weight measurements (at least 3 samples per coating) was employed in the following formula to obtain comparative results: 100× sample weight increase/uncoated $\mathrm{Al} 2024$ weight increase. This indicated the \% reduction obtained by each coating relative to the ice accreted on bare $\mathrm{Al} 2024$ coupons which is considered to be $100 \%$. 


\section{Results}

\subsection{Thickness and Roughness}

Figure 2 shows a scheme of the coating cross section and a top view of a coated specimen (Al coupon dimensions: $50 \mathrm{~mm} \times 50 \mathrm{~mm}$ ).

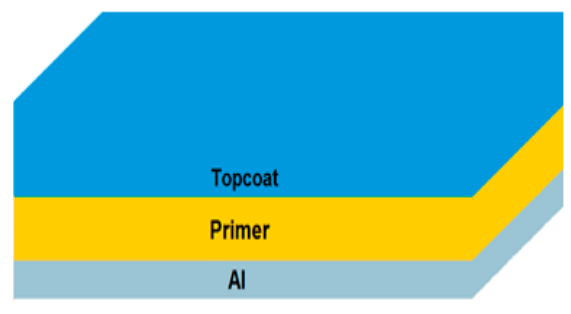

(a)

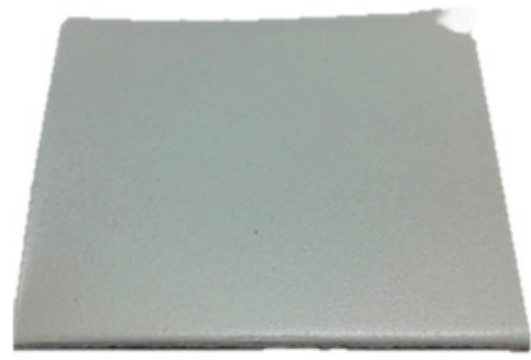

(b)

Figure 2. (a) Layer deposition diagram, (b) exemplary sample view.

The thickness of all the produced samples was measured twice, initially after deposition of the primer layer and then after the modified PUR coating was sprayed. The measured primer layer thickness was $25 \pm 3 \mu \mathrm{m}$ whereas that of the total coating system was $55 \pm 5 \mu \mathrm{m}$ for each of the produced samples. Only slight differences in coating thickness result from manual coating spraying.

The surface roughness was measured both qualitatively and quantitatively by means of an optical profilometer. Figure 3 presents 3D roughness profile images for non-modified coatings as well as coatings with 1 wt \% and $5 \mathrm{wt} \%$ of nano-silica.

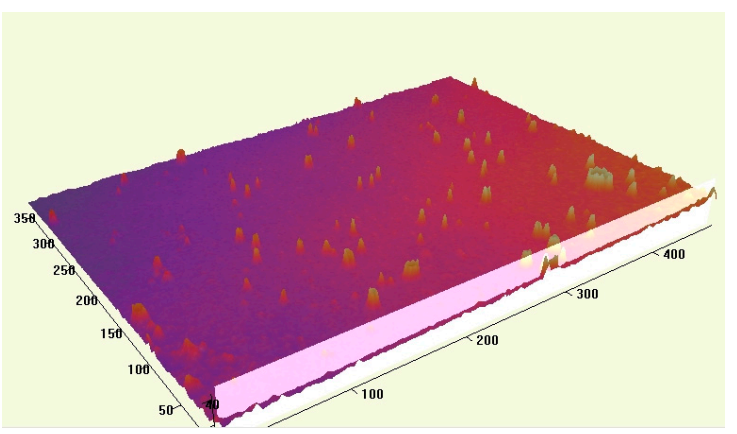

(a)

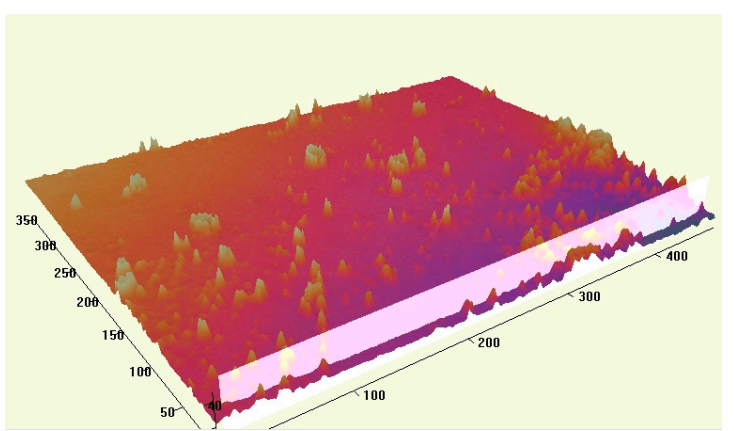

(b)

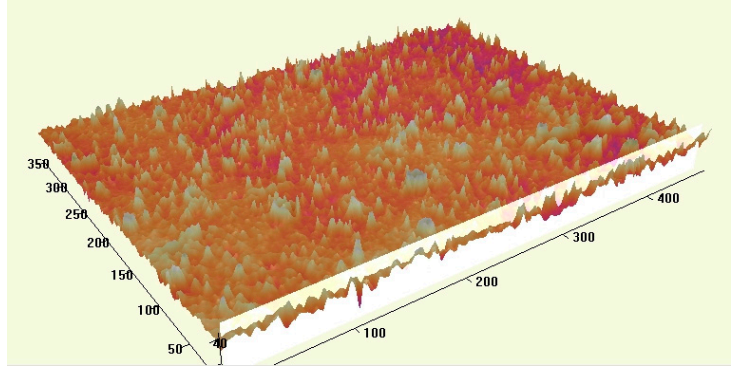

(c)

Figure 3. Three-dimensional (3D) roughness profile images for (a) reference coating, (b) Sample 1, (c) Sample 2. Vertical scales in panels (a-c) indicate $40 \mu \mathrm{m}$.

The scanned area was $450 \mu \mathrm{m} \times 350 \mu \mathrm{m}$. As can be observed in Figure 3a, the roughness profile is uniform with only some irregularities in the case of the reference sample since the coating does not 
contain nano-silica. On the other hand, upon increasing the nano-silica content, the profiles become rougher. Figure $3 \mathrm{~b}$ illustrates the roughness profile for a sample with $1 \mathrm{wt} \%$ of nano-silica particles showing more irregularities but not a total coverage due to too low a nanoparticle content. In addition, a non-homogeneous distribution of nanoparticle agglomerates can be observed. Upon increasing the nano-silica content to $5 \mathrm{wt} \%$ in the case of Sample 2, a more uniform, rough surface can be observed. Such a structure should result in a high level of air trapping within the formed voids, thereby achieving higher hydrophobic properties [24-26].

Quantitative results were calculated for all investigated samples. Surface roughness can be described by various parameters but the most commonly used among them are average roughness $(R a)$ and maximum peak-to-peak roughness $(R z)$. Calculated parameters are presented in Table 2.

Table 2. Average $(R a)$ and maximum $(R z)$ roughness parameters of PUR coatings.

\begin{tabular}{ccccc}
\hline Sample & Ra $[\mu \mathrm{m}]$ & Std. Dev. & Rz $[\mu \mathrm{m}]$ & Std. Dev. \\
\hline ref & 0.087 & 0.0064 & 0.489 & 0.0362 \\
1 & 1.49 & 0.1941 & 8.48 & 0.5923 \\
2 & 3.20 & 0.2175 & 17.06 & 1.3392 \\
3 & 0.091 & 0.0073 & 0.461 & 0.0427 \\
4 & 3.36 & 0.2966 & 17.38 & 1.5284 \\
\hline
\end{tabular}

$R \mathrm{a}$ and $\mathrm{Rz}$ for the reference sample were 0.087 and $0.489 \mu \mathrm{m}$, respectively. These results indicate a low level of surface roughness due to no nano-silica in the coating composition. Upon increasing the nanoparticles content, the roughness increases. In the case of Sample 2, Ra and Rz were 3.2 and 17.06 $\mu \mathrm{m}$, respectively.

As shown in Table 2, the use of silane modifier DC88 did not influence the surface roughness. Ra and $R z$ parameters of Samples 3 and 4 are almost identical to samples without the chemical modifier.

\subsection{Surface Morphology}

The SEM was employed to observe the detailed microstructures for the samples with different nano-silica contents.

Figure $4 \mathrm{a}$ shows the smooth morphology of the non-modified PUR coating. Figure $4 \mathrm{~b}$ presents the morphology of sample with $1 \mathrm{wt} \%$ of nanoparticles, which exhibits a distribution of particles/agglomerates with large areas without them. Indeed, the $\mathrm{SiO}_{2}$ particle content is too low to cover whole surface, leading to partial exposure of pure PUR coating. Figure 4c shows the morphology of coating with $5 \mathrm{wt} \%$ of nano-silica, which is rough and more uniform. Increasing the nano-silica particle content led to complete coverage throughout the coating surface. In both samples with nano-silica, formation of agglomerates was detected (Figure $4 \mathrm{~d}, \mathrm{e}$ ).

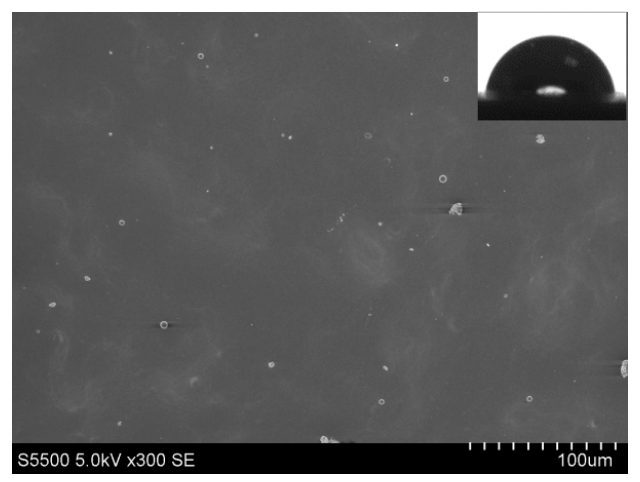

(a)

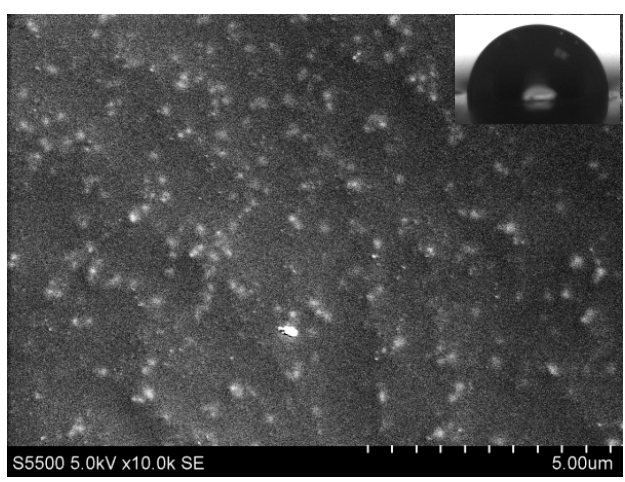

(b)

Figure 4. Cont. 


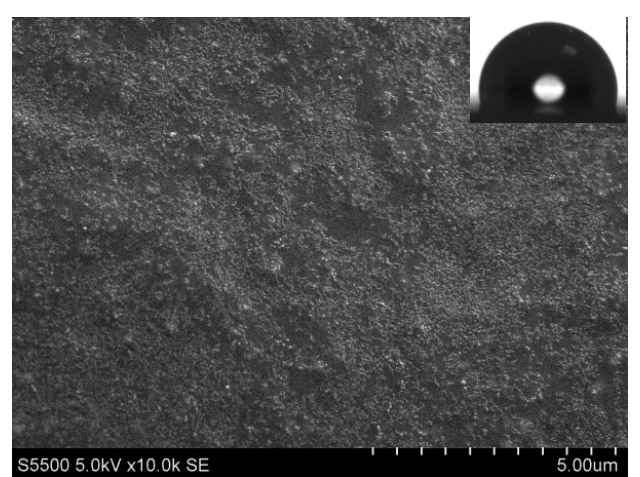

(c)

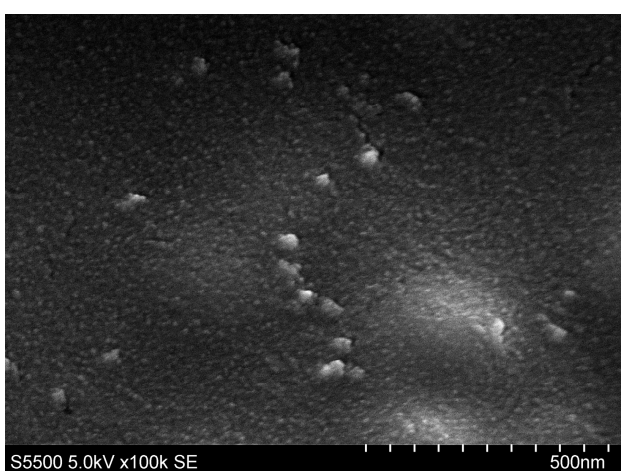

(d)

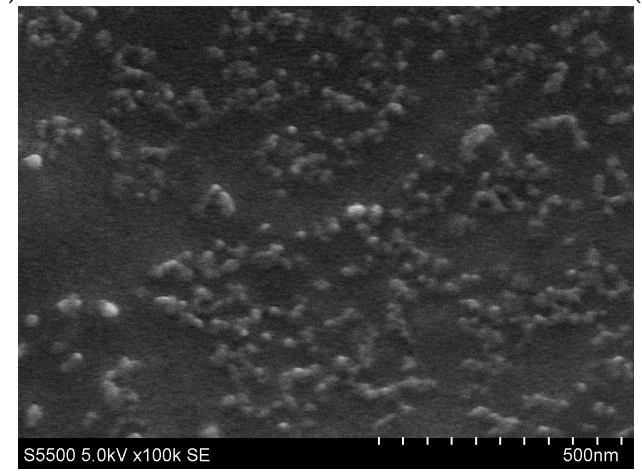

(e)

Figure 4. Scanning electron microscope (SEM) images of (a) reference sample, (b) Sample 1, (c) Sample 2, (d) Sample 1 agglomerates, (e) Sample 2 agglomerates. The insets are the corresponding water droplet profiles.

The XPS survey spectrum (Figure 5a) of Sample 4 reveals the characteristic signals of carbon (C 1s, Figure $5 b)$, nitrogen (N 1s, Figure 5c) and oxygen (O 1s, Figure 5d) at about 284.5, 399.7 and 532 $\mathrm{eV}$, respectively.

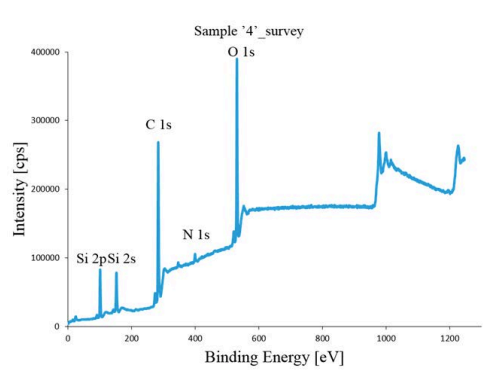

(a)

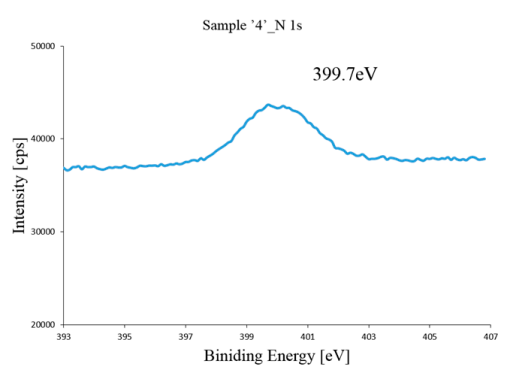

(c)

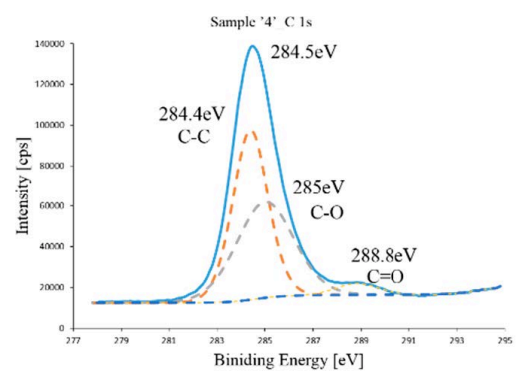

(b)

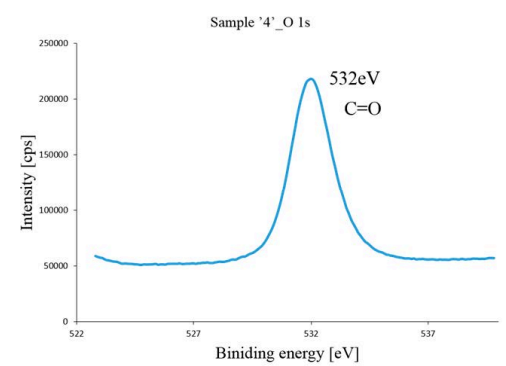

(d)

Figure 5. Cont. 


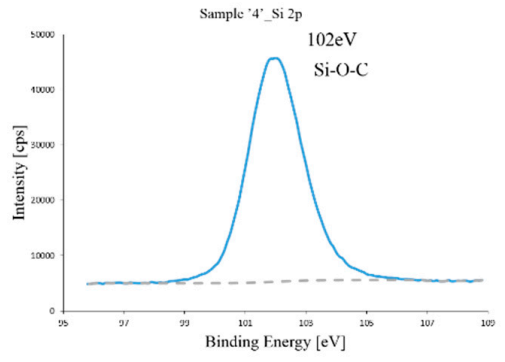

(e)

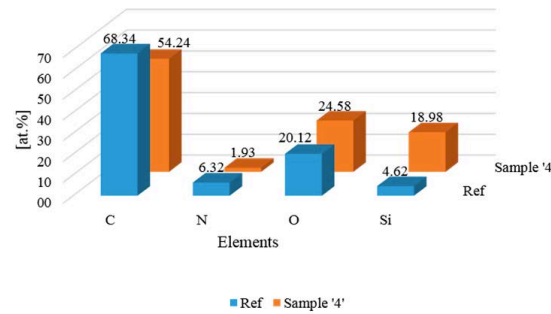

(f)

Figure 5. X-ray photoelectron spectroscopy (XPS) spectra: (a) survey spectrum of Sample 4, (b) C 1s of Sample 4, (c) N 1s of Sample 4, (d) O 1s of Sample 4, (e) Si 2p of Sample 4, (f) elemental analysis of Samples ref and $4^{\prime}$.

The existence of additional signals of silicon (Si $2 \mathrm{p}$ at about $102 \mathrm{eV}$ and $\mathrm{Si} 2 \mathrm{~s}$ at about $153 \mathrm{eV}$ ) indicates the incorporation of silicone. Figure $5 e$ shows the XPS spectra of Si $2 p$ on the surface of Sample 4. The peak at $102.004 \mathrm{eV}$ is the binding energy of $\mathrm{Si}-\mathrm{O}-\mathrm{C}$. The $\mathrm{Si}-\mathrm{O}-\mathrm{C}$ bond corresponds to trimethoxysilane groups which have survived during the coating formation [27]. Additionally, by employing the elemental analyser (Figure 5f), the atom ratios of C, N, O and $\mathrm{Si}$ in Samples Ref and 4 are defined as $68.3 ; 6.32 ; 20.12$ and 4.62 at $\%$ and $54.24 ; 1.93 ; 24.58$ and 18.98 at \%, respectively. The analysis indicates that the level of Si in Sample 4 is four time higher than in the reference which confirms presence of silicone groups on the sample surface.

\subsection{Wettability Behaviour}

Surface wettability of the as-prepared coatings was investigated by water contact angle measurements. SCA, ACA, RCA, CAH and SA values for all manufactured samples are shown in Table 3.

Table 3. Wetting properties for the reference and nanocomposite coatings.

\begin{tabular}{|c|c|c|c|c|c|c|c|c|c|}
\hline Sample & $\mathrm{SCA}\left[{ }^{\circ}\right]$ & Std. Dev. & $\mathrm{ACA}\left[{ }^{\circ}\right]$ & Std. Dev. & $\operatorname{RCA}\left[{ }^{\circ}\right]$ & Std. Dev. & $\mathrm{CAH}\left[{ }^{\circ}\right]$ & SA $\left[{ }^{\circ}\right]$ & Std. Dev. \\
\hline ref & 84 & 1.48 & 86 & 0.75 & 47 & 0.83 & 39 & 87 & 1.21 \\
\hline 1 & 94 & 0.67 & 98 & 0.93 & 63 & 1.21 & 35 & 67 & 1.35 \\
\hline 2 & 104 & 1.34 & 107 & 0.61 & 76 & 0.43 & 31 & 62 & 0.98 \\
\hline 3 & 105 & 0.71 & 108 & 1.03 & 79 & 1.09 & 29 & 39 & 1.52 \\
\hline 4 & 114 & 1.23 & 118 & 0.77 & 93 & 0.84 & 25 & 35 & 0.87 \\
\hline
\end{tabular}

As already mentioned, polyurethanes are hydrophilic polymers due to polar groups present in their chain structure [28]. In fact, SCA and SA of the reference coating were $84^{\circ}$ and $87^{\circ}$, respectively, confirming the hydrophilic behaviour of this coating. The use of nano-silica only caused a slight improvement of hydrophobic properties despite the differences in roughness. This could be due to a higher specific area caused by the presence of the particles. A superhydrophobic behaviour was still not achieved $\left(\mathrm{SCA}>150^{\circ}\right)$.

The wetting behaviour is influenced not only by the surface roughness but also by the surface chemical composition $[29,30]$. Indeed, the presence of the silane modifier DC 88 , which was added to reduce the surface energy also improved the reference coating's wetting properties reaching a SCA very similar to that of the coating containing $5 \mathrm{wt} \%$ of nano-silica $\left(105^{\circ}\right.$ and $104^{\circ}$, respectively). The presence of silane groups impacts the hydrophobic behaviour by shielding the polar groups of PUR from interaction with water [31,32]. Indeed, coating with both the DC88 modifier and $5 \mathrm{wt} . \%$ nano-silica caused further improvement of the hydrophobic behaviour of the coating. This observation confirms that both factors, surface roughness and chemical composition, play a significant role on the final hydrophobic properties of the coating, as expected. 


\subsection{Frosting/Defrosting Cycles}

SCAs were measured between frosting/defrosting cycles in order to achieve a better understanding of the durability of the wetting behaviour after exposure to icing conditions. Figure 6 shows the variation of the SCAs of the reference as well as Samples 2 and 4 as they were repeatedly subjected to frosting/defrosting.

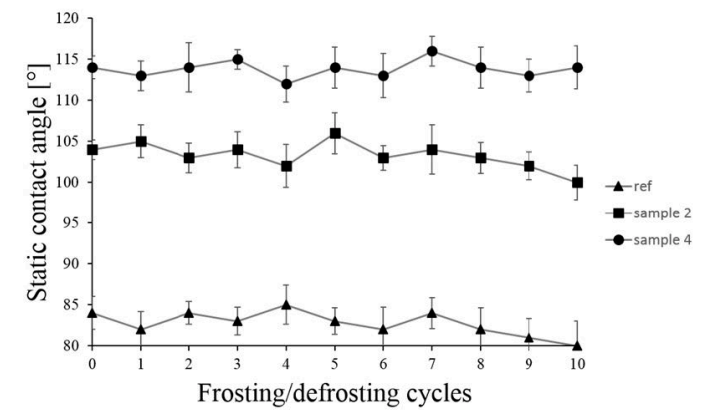

Figure 6. SCA as a function of frosting/defrosting cycles.

Noteworthy in Figure 6 is the fact that the wetting properties of Sample 4 with both nano-silica and the DC88 modifier were maintained after the 10 cycles. On the other hand, the SCA values of the reference and Sample 2 (with only nano-silica) started to decrease after seven cycles. This slight deterioration can be attributed to a gradual damage of the rough structures of the sample surface [33]. In the case of Sample 4, such deterioration was not observed, indicating that the silane modifier induces a more stable improvement of both hydrophobic and anti-icing properties of the coating.

\subsection{Coating Adhesion}

Most of the scientific papers dealing with hydrophobic/icephobic coatings focus on modification of specific functional properties, without taking into account the fact that such modifications can deteriorate other parameters like durability, thermal stability, adhesion to substrate, etc. In this work, the effect of coating modification by adding nano-silica and the silane DC88 additive on the adhesion to the $\mathrm{Al} 2024$ substrate was investigated by using a scratch tester. The test was performed by applying a linearly increasing load, from 1 to up to $20 \mathrm{~N}$ until coating failure occurred. Five scratches were performed on each sample to provide a measurement the coating-substrate adhesion (defined as the lowest load at which the coating detached from the substrate). Each scratch was examined with an optical microscope. For all samples it was noted that the PUR coating never once separated from the primer layer indicating very good adhesion between both layers. The critical loads determined are shown in Figure 7.

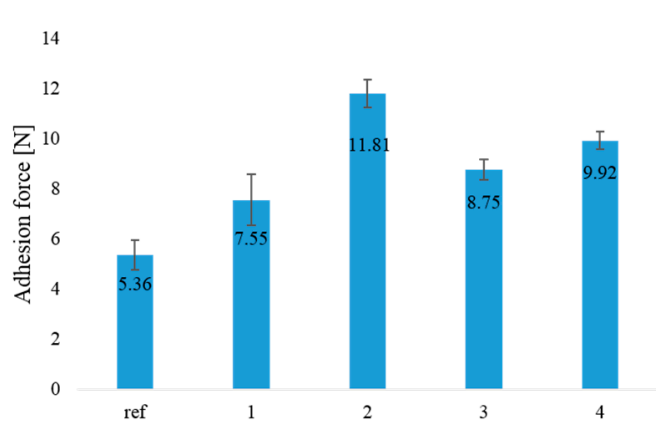

Figure 7. Adhesion critical forces of PUR coatings. 
The reference sample exhibited the lowest adhesion, indicating that none of the coating modifications deteriorated the adhesion on $\mathrm{Al} 2024$. Addition of $5 \mathrm{wt} \%$ of nano-silica, on the other hand, increased the adhesion 2 fold in comparison to the reference coating.

\subsection{Ice Accretion Test}

The samples were exposed to glaze ice, which is typically formed in clouds. The test conditions to obtain it were described in Section 2.3. Glaze ice is transparent or translucent and is formed by drops of supercooled water that, when hitting the surface, do not fully freeze immediately. It is a denser and harder ice, more difficult to break and usually more transparent than rime ice and, therefore, more difficult to detect, implying higher danger. It is also more adherent (and therefore harder to detach) and modifies the profile of the components on which it forms to a greater extent, affecting the corresponding aerodynamics [34]. The accreted ice \% relative to the uncoated reference is presented in Table 4 .

Table 4. Ice accretion test results.

\begin{tabular}{ccc}
\hline Sample & Accreted Ice [\%] & Std. Dev. \\
\hline Bare Al & 100 & - \\
ref & 85 & 1.34 \\
1 & 88 & 0.68 \\
2 & 92 & 1.21 \\
3 & 83 & 1.55 \\
4 & 77 & 1.62 \\
\hline
\end{tabular}

The lower the percentage, the less ice was accreted. Although the differences are very small, the results show a correlation between ice accretion and the roughness as well as the SCA for the samples without the silane DC88 modifier; the higher the roughness and the SCA, the higher the ice accretion. Moreover, the silane additive (Sample 3) on its own did not significantly affect the behaviour of the reference PUR coating. However, the combined effect of the nanoparticles (Sample 4) and the additive caused a marked decrease in ice accretion and resulted in the best coating behaviour, in particular when comparing it with Sample 2, modified with $5 \mathrm{wt} \%$ of nano-silica but without the additive. This demonstrates that the chemical composition of the surface (therefore the surface energy) plays a very important role in icing mitigation.

Despite not exhibiting superhydrophobic performance, the anti-icing behaviour of Sample 4 was significantly better than that of the reference coating, confirming the lack of direct correlation between the hydrophobic properties and the anti-icing behaviour. In addition, the best-performing materials (Sample 3: 83\%; Sample 4: 77\%) had totally different surface roughness parameters (Ra: 0.091 and 3.36 $\mu \mathrm{m}$, respectively) which indicates that in this case the composition and surface energy properties seem to have a higher effect than microstructural aspects.

\section{Conclusions}

Hydrophobic nanocomposite coatings were manufactured based on a polyurethane topcoat and modified by adding nano-silica particles and a silane modifier. The main goal was to decrease the wetting behaviour of hydrophilic polyurethane coating through addition of nano-silica and modification of the chemical composition by silane-based compounds. Using scanning electron microscopy and optical profilometry, it was found that addition of $1 \mathrm{wt} \%$ of nano-silica did not result in total coverage of the surface by nano-silica particles and only slightly improved the hydrophobic behaviour. Results of the scratch tests performed demonstrated that the modifications do not worsen coating adhesion to the aluminium 2024 substrate. It was also confirmed that the surface chemical composition influences the anti-icing properties of the coating and, to some degree, also the wetting behaviour. The results also showed that the anti-icing behaviour does not necessarily correlate with surface roughness and the 
materials' wetting properties. However, a synergistic effect was observed on the coating combining the silane additive with $5 \mathrm{wt} . \%$ of nano-silica, which exhibited high roughness and showed ice accretion reduction relative to the polyurethane reference.

Author Contributions: Conceptualization, B.P.; methodology, B.P. and R.K.; investigation, B.P., R.K., J.M., P.G. and A.B. (Ana Borras); writing-original draft preparation, B.P. and J.M.; writing-review and editing, A.B. (Anna Boczkowska) and A.A.; supervision, R.K. and A.B. (Anna Boczkowska); project administration, R.K.

Funding: This work was part of the project "Super-Ice-Phobic Surfaces to Prevent Ice Formation on Aircraft" supported by the European Council (Grant No. 690819).

Conflicts of Interest: The authors declare no conflict of interest.

\section{References}

1. Antonini, C.; Innocenti, M.; Horn, T.; Marengo, M.; Amirfazli, A. Understanding the effect of superhydrophobic coatings on energy reduction in anti-icing systems. Cold Reg. Sci. Technol. 2011, 67, 58-67. [CrossRef]

2. Boreyko, J.B.; Collier, C.P. Delayed frost growth on jumping-drop superhydrophobic surfaces. ACS Nano 2013, 7, 1618-1627. [CrossRef] [PubMed]

3. Brouwers, E.W.; Peterson, A.A.; Palacios, J.L.; Centolanza, L.R. Ice adhesion strength measurements for rotor blade leading edge materials. In Proceedings of the 67th American Helicopter Society International Annual Forum, Virginia Beach, VA, USA, 3-5 May 2011.

4. Boinovich, L.B.; Emelyanenko, A.M.; Emelyanenko, K.A.; Modin, E.B. Modus operandi of protective and anti-icing mechanisms underlying the design of longstanding outdoor icephobic coatings. ACS Nano 2019, 13, 4335-4346. [CrossRef] [PubMed]

5. Tarquini, S.; Antonini, C.; Amirfazli, A.; Marengo, M.; Palacios, J. Investigation of ice shedding properties of superhydrophobic coatings on helicopter blades. Cold Reg. Sci. Technol. 2014, 100, 50-58. [CrossRef]

6. Ky, P. Annual Safety Review 2017; EASA: Cologne, Germany, 2017; p. 12.

7. Coffman, H.J. Helicopter Rotor Icing Protection Methods. J. Am. Helicopter Soc. 1987, 32, 34-39. [CrossRef]

8. Itagaki, K. Self-Shedding from High-Speed Rotors. In Mechanical Ice Release Processes; US Army Corps of Engineers, Cold Regions Research \& Engineering Laboratory: Hanover, NH, USA, 1983.

9. Mishchenko, L.; Hatton, B.; Bahadur, V.; Taylor, J.A.; Krupenkin, T.; Aizenberg, J. Design of ice-free nanostructured surfaces based on repulsion of impacting water droplets. ACS Nano 2010, 4, 7699-7707. [CrossRef]

10. Karmouch, R.; Coude, S.; Abel, G.; Ross, G.G. Icephobic PTFE coatings for wind turbines operating in cold climate conditions. In Proceedings of the 2009 IEEE Electrical Power \& Energy Conference, Montreal, QC, Canada, 22-23 October 2009.

11. Kulinich, S.A.; Farzaneh, M. Ice adhesion on super-hydrophobic surfaces. Appl. Surf. Sci. 2009, 255, 8153-8157. [CrossRef]

12. Seyfi, J.; Jahari, S.H.; Khonakdar, H.A.; Sadeghi, G.M.M.; Zohuri, G.; Hejazi, I.; Simon, F. Fabrication of robust and thermally stable superhydrophobic nanocomposite coatings based on thermoplastic polyurethane and silica nanoparticles. Appl. Surf. Sci. 2015, 347, 224-230. [CrossRef]

13. Menini, R.; Farzaneh, M. Advanced Icephobic Coatings. J. Adhes. Sci. Technol. 2011, 25, 971-992. [CrossRef]

14. Work, A.; Lian, Y. A critical review of the measurement of ice adhesion to solid substrates. Prog. Aerosp. Sci. 2018, 98, 1-26. [CrossRef]

15. Koch, K.; Bhushan, B.; Jung, Y.C.; Barthlott, W. Fabrication of artificial Lotus leaves and significance of hierarchical structure for superhydrophobicity and low adhesion. Soft Matter 2009, 5, 1386-1393. [CrossRef]

16. Koch, K.; Bhushan, B.; Barthlott, W. Diversity of structure, morphology and wetting of plant surfaces. Soft Matter 2008, 4, 1943-1963. [CrossRef]

17. Hejazi, I.; Sadeghi, G.M.M.; Seyfi, J.; Jafari, S.H.; Khonakdar, H.A. Self-cleaning behavior in polyurethane/silica coatings via formation of a hierarchical packed morphology of nanoparticles. Appl. Surf. Sci. 2016, 368, 216-223. [CrossRef] 
18. El Dessouky, W.I.; Abbas, R.; Sadik, W.A.; El Demerdash, A.G.M.; Hefnawy, A. Improved adhesion of superhydrophobic layer on metal surfaces via one step spraying method. Arab. J. Chem. 2017, 10, 368-377. [CrossRef]

19. Lazauskas, A.; Guobiene, A.; Prosycevas, I.; Baltrusaitis, V.; Grigaliunas, V.; Narmontas, P.; Baltrusaitis, J. Water droplet behavior on superhydrophobic $\mathrm{SiO}_{2}$ nanocomposite films during icing/deicing cycles. Mater. Charact. 2013, 82, 9-16. [CrossRef]

20. Król, P.; Król, B.; Kozakiewicz, J.; Zapotoczny, S.; Pilch-Pitera, B.; Kozdra, S. Composites prepared from polyurethanes modified with silicone-acrylic nanopowders. Prog. Org. Coat. 2015, 81, 72-79. [CrossRef]

21. Verma, G.; Kaushik, A.; Ghosh, A.K. Comparative assessment of nano-morphology and properties of spray coated clear polyurethane coatings reinforced with different organoclays. Prog. Org. Coat. 2013, 76, 1046-1056. [CrossRef]

22. West, J.O.F.; Critchlow, G.W.; Lake, D.R.; Banks, R. Development of a superhydrophobic polyurethane-based coating from a two-step plasma-fluoroalkyl silane treatment. Int. J. Adhes. Adhes. 2016, 68, 195-204. [CrossRef]

23. Tang, Y.; Yang, J.; Yin, L.; Chen, B.; Tang, H.; Liu, C.; Li, C. Fabrication of superhydrophobic polyurethane/MoS 2 nanocomposite coatings with wear-resistance. Colloids Surf. A Physicochem. Eng. Asp. 2014, 459, 261-266. [CrossRef]

24. Kosak Söz, C.; Yilgör, E.; Yilgör, I. Influence of the average surface roughness on the formation of superhydrophobic polymer surfaces through spin-coating with hydrophobic fumed silica. Polymer 2015, 62, 118-128. [CrossRef]

25. Zou, M.; Beckford, S.; Wei, R.; Ellis, C.; Hatton, G.; Miller, M.A. Effects of surface roughness and energy on ice adhesion strength. Appl. Surf. Sci. 2011, 257, 3786-3792. [CrossRef]

26. Bharathidasan, T.; Kumar, S.V.; Bobji, M.S.; Chakradhar, R.P.S.; Basu, B.J. Effect of wettability and surface roughness on ice-adhesion strength of hydrophilic, hydrophobic and superhydrophobic surfaces. Appl. Surf. Sci. 2014, 314, 241-250. [CrossRef]

27. Zhao, H.; Hao, T.H.; Hu, G.H.; Shi, D.; Huang, D.; Jiang, T.; Zhang, Q.C. Preparation and characterization of polyurethanes with cross-linked siloxane in the side chain by sol-gel reactions. Materials 2017, 10, 247. [CrossRef] [PubMed]

28. Luo, Z.; Hong, R.Y.; Xie, H.D.; Feng, W.G. One-step synthesis of functional silica nanoparticles for reinforcement of polyurethane coatings. Powder Technol. 2012, 218, 23-30. [CrossRef]

29. Shirtcliffe, N.J.; McHale, G.; Atherton, S.; Newton, M.I. An introduction to superhydrophobicity. Adv. Colloid Interface Sci. 2010, 161, 124-138. [CrossRef]

30. Furstner, R.; Barthlott, W.; Neinhuis, C.; Walzel, P. Wetting and self-cleaning properties of artificial superhydrophobic surfaces. Langmuir 2005, 21, 956-961. [CrossRef]

31. Hang, T.T.X.; Dung, N.T.; Truc, T.A.; Duong, N.T.; Truoc, B.V.; Vu, P.G.; Hoang, T.; Thanh, D.T.M.; Olivier, M.G. Effect of silane modified nano ZnO on UV degradation of polyurethane coatings. Prog. Org. Coat. 2015, 79, 68-74. [CrossRef]

32. Farhadi, S.; Farzaneh, M.; Simard, S. On stability and ice-releasing performance of nanostructured fluoro-alkylsilane-based superhydrophobic Al alloy2024 surfaces. Int. J. Theor. Appl. Nanotechnol. 2012, 1, 38-45. [CrossRef]

33. Farhadi, S.; Farzaneh, M.; Kulinich, S.A. Anti-icing performance of superhydrophobic surfaces. Appl. Surf. Sci. 2011, 257, 6264-6269. [CrossRef]

34. Liao, R.; Zuo, Z.; Guo, C.; Zhuang, A.; Zhao, X.; Yuan, Y. Anti-icing performance in glaze ice of nanostructured film prepared by RF magnetron sputtering. Appl. Surf. Sci. 2015, 356, 539-545. [CrossRef]

(C) 2019 by the authors. Licensee MDPI, Basel, Switzerland. This article is an open access article distributed under the terms and conditions of the Creative Commons Attribution (CC BY) license (http://creativecommons.org/licenses/by/4.0/). 